\title{
MATHEMATICAL DEGRADATION MODEL FOR TRANSCRIPTIONAL REGULATION OF BRCA1
}

\author{
S.Lakshmi ${ }^{1}$ and K.Majella Jenvi Ignatia ${ }^{2}$
}

\begin{abstract}
In this paper we study the degradation processes of the altered BRCA1 transcription using Generalized Birth and death process and Hazard rate function $\mathrm{H}(\mathrm{u})$ in order to find the bound and mean life time for the breast cancer affected patients. The results available in literature show that Estrogen kinetics inhibits most vigorously in breast cancer and dismisses CtBP which leads to break the linkages in tumor cells and control. The break through is that Estrogen kinetics inhibits most vigorously in breast cancer and dismisses CtBP which leads to break the linkages in tumor cells and control rather than Trichostatin and its kinetics as Estrogen has most effect on breast cancer. We have also shown that Estrogen has better effect rather than Trichostatin by considering hazard rate and mean life time of BRCA1 transcription. Mean life time provided by TrichostatinA and TrichostatinA plus cycloheximide behaves as the bound for mean life time provided by Estrogen and Estrogen plus cycloheximide for Trefoil factor1. Also we state that NBR2 promoters have the same effects between Trichostatin kinetics and Estrogen kinetics The study of Estrogen and Trichostatin in this biological criteria which is considered for our study only concentrated on behavior of Estrogen effects and concluded that E2+CH substantially induces BRCA1 neither TFF1 nor NBR2. We extend this result that BRCA1 altered transcription has better inhibition in HDAC than untreated control and the Estrogen kinetics inhibits most vigorously in breast cancer and dismisses $\mathrm{CtBP}$ which leads to break the linkages in tumor cells and control rather than Trichostatin. Further we extend this study by developing Degradation model from Birth and Death process and derive the Reliability function, Hazard rate average for the same biological system. Therefore we can find the influence of the hormone and its kinetics on the three different secretary proteins.
\end{abstract}

Keywords - Reliability function R(t), Hazard rate average H(u), Breast cancer gene1 (BRCA1), Trefoil Factor 1 (TFF1), Neibor of BRCA1 gene 2 (NBR2)

\section{INTRODUCTION}

Estrogen is the most important "female" hormone. Its deficiency occurs when Estrogen level lowers in the body. Deficiency usually occurs during menopause, but sometimes it can occur even before the menopausal stage too. This disturbs the menstrual cycle, which leads to more disorders. Some women may also have early menopause that is before the age of 40. This also results in low Estrogen levels in the body. Deficiency of this hormone is considered to be a very serious concern and needs be treated by natural foods or dietary supplements. Other things that may cause Estrogen deficiency in women includes Eating disorders, Pituitary

\footnotetext{
${ }^{1}$ Kundavai Naachiyaar Govt Arts College for women, Tanjore, Tamil Nadu, India.

${ }^{2}$ Department of Mathematics Bharathiar University, Coimbatore, Tamil Nadu, India.
} 
diseases, Low body fat, Menopause, Thyroid disorders, Genetic diseases, Breast changes, Breast cancer. Breast cancer is the second leading cause of death in women worldwide and the primary cause of death from cancer in women aged in between $20-59^{[8]}$. Individuals harboring germ line mutations in the breast cancer susceptibility gene BRCA1 carry an $80 \%$ lifetime risk of developing breast cancer ${ }^{[7]}$. Though very few cases of non inherited sporadic forms of breast cancer are associated with mutation in BRCA1, 40\% of these tumors have a deficiency in BRCA1 expression ${ }^{[13]}$. Because the majority of these cases do not depict the hypermethylation of the BRCA1 promoter, a growing consensus has emerged suggesting that a large percentage of sporadic, non inherited breast cancers are associated with altered transcriptional regulation of the BRCA1 gene ${ }^{[13,19]}$.

TrichostatinA (TSA), an antifungal antibiotic with cytostatic and differentiating properties in mammalian cell culture, is a potent and specific inhibitor of histone deacetylase (HDAC) activity. The purpose of this study was to evaluate the antiproliferative and HDAC inhibitory activity of TSA in vitro in human breast cancer cell lines. Therefore the potent dosedependent antitumor activity of TSA against breast cancer in vitro and in vivo, strongly supporting HDAC as a molecular target for anticancer therapy in breast cancer ${ }^{[17]}$. Estrogens play a major role in promoting the proliferation of both the normal and the neoplastic breast epithelium. Their role as breast carcinogens has long been suspected and recently confirmed by epidemiological studies. Hence our attention is focused towards the Estrogen kinetics and this study mainly focuses to expose the importance of Estrogen rather than Trichostatin

The rest of the paper is organized as follows. Proposed degradation model for the biological system is explained in section II. Results and implementations are presented in section III. Concluding remarks are given in section IV.

\section{PROPOSED STUDY}

A. Biological system -

$\mathrm{CtBP}(\mathrm{C}$-terminal-binding protein) is a homodimer or heterodimer of $\mathrm{CtBP} 1$ and $\mathrm{CtBP} 2$ that assembles with a diverse array of factors that regulate chromatin structure. These include the histone deacetylases deacetylases (HDACs) HDAC1 and HDAC2, the histone acetyltransferases $\mathrm{p} 300$ and $\mathrm{CtBP}$, and the histone methyltransferase $\mathrm{G} 9 \mathrm{a}^{[3]}$. CtBP can antagonize the expression of multiple tumor suppressors. Most notably, CtBP contains a binding site for NADH that regulates its ability to dimerize, thus establishing CtBP as an important nuclear sensor of cellular metabolic status ${ }^{[6,20]}$. We demonstrate here that CtBP assembles at the BRCA1 promoter as part of a dynamic, multicomponent co-repressor complex containing p130, BRCA1 and HDAC1 that represses local histone acetylation at the BRCA1 promoter and BRCA1 transcription. Disruption of this complex by Estrogen stimulation and/or changes in $\mathrm{NAD}^{+} / \mathrm{NADH}$ ratio leads to CtBP dismissal, HDAC1 eviction, increased histone acetylation and subsequent increased BRCA1 transcription from the BRCA1 promoter. These observations define a direct link between cellular metabolic status and the expression of BRCA1 and suggest that caloric intake may selectively influence tumor suppressor function in mammary tissues. BRCA1 itself can form complexes with HDACs either directly or through interactions involving the CtBP - interacting protein ${ }^{[5]}$.Estrogen and its kinetics have better effect on breast cancer cells with trefoil factor 1 (TFF1) protein ${ }^{[11]}$.

The mechanism by which CtBP can control BRCA1 transcription involves direct regulation of the local chromatin marks within the BRCA1 bidirectional promoter by preventing histone $\mathrm{H} 3$ and $\mathrm{H} 4$ acetylation through $\mathrm{HDAC} 1$ recruitment. If the key regulatory step in this process is histone acetylation, then HDAC inhibition would probably lead to BRCA1 transcriptional induction. This is indeed the case, because incubation of MCF-7 with TrichostatinA produces a rapid induction of BRCA1 transcription that occurs much earlier than Estrogen stimulation for both mature and nascent RNA transcripts, see Figure $1^{[9]}$. Also, like Estrogen, TSA induction is inhibited by treatment with protein synthesis inhibitors. Notably, the influence of 
HDAC inhibition is both promoter-specific and directional because neither TFF1 nor NBR2 are substantially induced by HDAC inhibition, see Figure 1. Moreover, both Estrogen treatment and HDAC inhibition produce nearly identical increases in chromatin

Accessibility at the BRCA1 promoter compared with the untreated control. As authors expected, HDAC inhibition with TSA leads to substantially increased histone $\mathrm{H} 3$ and $\mathrm{H} 4$ acetylation at the BRCA1 promoter in addition to some alteration in the assembly of HDAC1, BRCA1 and p130, but does not substantially influence the assembly of CtBP, E2F1 and p300. These differences suggest that although histone acetylation is a major target, additional protein or factor acetylation may also have a role in the stability of the co-repressor complexes assembled at the BRCA1 promoter, see Figure 1. Finally, loss of p300 by RNAi depletion renders the BRCA1 promoter unresponsive to TSA treatment and blocks the increase in promoter-proximal histone acetylation, thus demonstrating that p300 is primarily responsible for the positive influence of HDAC inhibition on histone acetylation and transcription at the BRCA1 promoter. Estrogen treatment is a major form of mitogenic stimulation that increases cellular proliferation in normal mammary tissues and enhances cell cycle entry in breast-derived cell lines like MCF-7. The elevated respiration associated with increased proliferation causes the $\mathrm{NAD}^{+} / \mathrm{NADH}$ ratio to rise to meet the increased energy demand due to surges in protein synthesis and DNA replication ${ }^{[12]}$. Although hypoxia blocks BRCA1 transcription in addition to other factors important in the response to DNA damage $^{[10]}$, its influence on Estrogen-regulated induction of BRCA1 has not been explored.
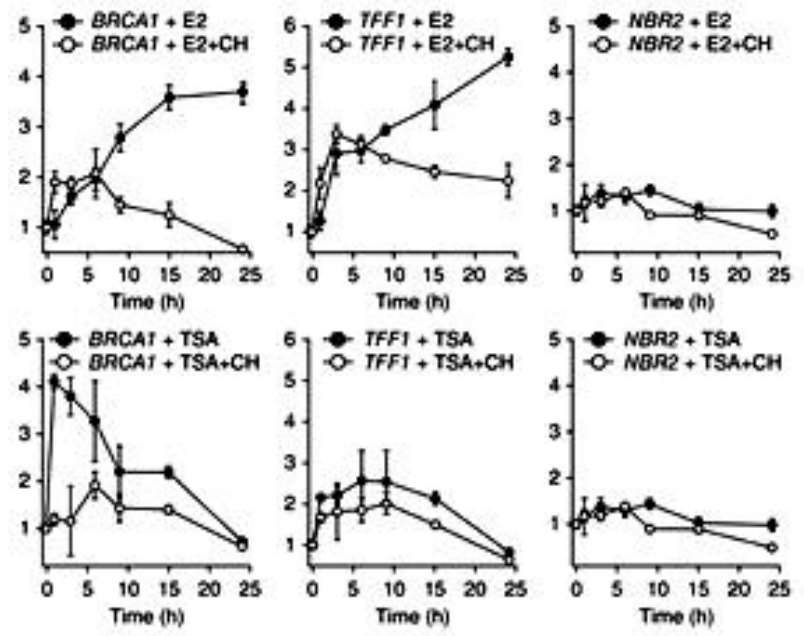

Figure 1: TSA and Estradiol (E2)-induced activation of BRCA1 by increasing p300-dependent histone acetylation at the BRCA1 promoter.

Time course of TFF1, NBR2 and BRCA1 expression in MCF-7 cells treated 0-24 h with either Estradiol (E2), Estradiol plus cycloheximide (E2 + CH, $\left.10 \mu \mathrm{g} \mathrm{ml}^{-1}\right)$, TrichostatinA (500 $\mathrm{ng} \mathrm{ml}^{-1}$ ), or TrichostatinA plus cycloheximide (TSA $\left.+\mathrm{CH}\right)$.

The study of Estrogen and Trichostatin in this biological criterion was only concentrated on behavior of Estrogen effects. Further we extend this study by developing Degradation model from Birth and Death process and derive the Reliability function, Hazard rate average for the same biological system. Therefore we can find the influence of the hormone and its kinetics on the three different secretary proteins.

\section{B. Degradation model for the biological system -}

At present time, the study of degradation process is an issue of remarkable consideration because it is one of the modern mathematical fields that arise from modeling of mechanical and biological systems. Here we propose a model for our proposed study. In this paper we deal with the generalized Birth \& Death process as a model for degradation and aging process 
for biological objects is proposed. In earlier studies the model of complex hierarchical system and methods for calculation of the stationary and non stationary characteristics under special restoration strategies were proposed ${ }^{[2]}$. Controllable fault-tolerance systems which may be regarded as ageing control models were discussed ${ }^{[14]}$. Recurrency of the embedded chain of the non-decomposable semi markov process without instantaneous states is one of the possible conditions for its non degeneracy ${ }^{[15]}$. The conditional state probabilities for given object's life period and their limiting values when $\mathrm{t} \rightarrow \infty$ are calculated. The variation of the model parameters allows to consider various problems of aging and degradation control. Finally our application part illustrates our approach.

Most of up-to-data complex biological objects with sufficiently high organization during their life period pass over different states of evolution and existence. From reliability point of view those states can be divided into three groups; the states of normal functioning, the dangerous (degradation) states and the failure states.

In the simplest case, if the nature of the degradation process allows to completely order the states to admit the transition possibilities only neighboring states it can be modeled by the process of B \& D type.

Let us consider a system with only three states, which can be considered as an example of the aggregated states model ${ }^{[1,16]}$, where all states of each group: normal functioning $\mathrm{N}$, degradation D, and failure F are joined into one. Suppose for the simplicity that the failures arise in accordance with the Poisson flow, but the retrieval of control times are distributed with c.d.f. $\mathrm{B}(\mathrm{x})$ and the hazard rate $\beta(\mathrm{x})$. Moreover suppose that the direct transition from normal state into the failure state are also possible with intensity $\gamma$. Sufficiency of the strong regularity of the semi-Markov process for its nondegeneracy is proved through examples ${ }^{[4]}$.

In accordance with the given transition graph the Kolmogorov's system of differential equations for system states probabilities has the form

$$
\left\{\begin{array}{l}
\frac{d_{\pi} N(t)}{d t}=-(\lambda+\gamma) \pi N(t)+\int_{0}^{t} \beta(x) \pi_{D}(t, x) d x \\
\frac{\partial \pi_{D}(t, x)}{\partial t}+\frac{\partial \pi_{D}(t, x)}{\partial x}=-[\mu+\beta(x)] \pi_{D}(t, x), \\
\frac{d \pi_{F}(t)}{d t}=\gamma \pi_{N}(t)+\mu \pi_{D}(t, x)
\end{array}\right.
$$

with the initial and the boundary conditions

$$
\left\{\begin{array}{l}
\pi_{D}(t, 0)=\lambda \pi_{N}(t) \\
\pi_{N}(0)=1, \pi_{D}(0,0)=\pi_{F}(0)=0
\end{array}\right.
$$

The reliability function of the system is

$$
\mathrm{R}(\mathrm{t})=1-\pi_{F}(t)=1-\int_{0}^{t}\left[\left[\gamma \pi_{N}(u)+\mu \pi_{D}(u)\right] d u\right]
$$

where the functions $\pi_{N}(t)$ and $\pi_{D}(t, x)$ are the solutions of the two first equations of the system (1) and

$$
\pi_{D}(t)=\int_{0}^{t} \pi_{D}(t, x) d x
$$

where the function $g_{D}(t)$ is determined form the boundary condition (2) It gives

$$
\pi_{D}(t, x)=\lambda \pi_{N}(t-x) e^{-\mu x}(1-B(x))
$$

Substitution of this solution into the first equation of the system (10) gives the following equation 


$$
\frac{d \pi_{N}(t)}{d t}=-(\lambda+\gamma) \pi_{N}(t)+\lambda \int_{0}^{t} \beta(x) \pi_{N}(t-x) e^{-\mu x}(1-B(x)) d x
$$

The best method for its solution is a LT approach. In the terms of LT with the initial condition (2) and equation (6) after the usual order of integration changing takes the form.

$s \tilde{\pi}_{N}(s)-1=-(\lambda+\gamma) \tilde{\pi}_{N}(s)+\lambda \tilde{b}(s+\mu) \tilde{\pi}_{N}(s)$

where $\tilde{b}(s)=\int_{0}^{\infty} e^{-s t} b(t) d t \quad$ is a LT of the p.d.f $\mathrm{b}(\mathrm{t})$.

It follows form here that the solution of the last equation has a form.

$$
\tilde{\pi}_{N}(s)=[s+\gamma+\lambda(1-\tilde{b}(s+\mu))]^{-1}
$$

Where $\tilde{b}(s+\mu)$ is a monotone function ${ }^{[18]}$

Next the calculation of the LT $\tilde{\pi}_{D}(s)$ of the function $\pi_{D}(t)$, given by the equality (4) after substitution into it of the expression (7) gives.

$$
=\frac{\lambda \tilde{\pi}_{N}(s)(1-\tilde{b}(s+\mu))}{s+\mu}=\frac{\lambda(1-\tilde{b}(s+\mu))}{(s+\mu)(s+\gamma+\lambda(1-\tilde{b}(s+\mu))}
$$

At least for the LT $\tilde{\pi}_{F}(s)$ of the function $\pi_{F}(t)$ form the last of equations (10) one can find

$$
s \tilde{\pi}_{F}(s)=\gamma \tilde{\pi}_{N}(s)+\mu \tilde{\pi}_{D}(s)=\frac{\gamma(s+\mu)+\lambda \mu(1-\tilde{b}(s+\mu))}{(s+\mu)(s+\gamma+\lambda(1-\tilde{b}(s+\mu)))}
$$

\section{Mathematical model of CtBP dismissal process by TSA and estrogen kinetics}

Generalized Birth and Death processes which are special class of semi morkov processes are introduced for Degradation modeling of Estrogen kinetics in BRCA1 promoters influence $\mathrm{CtBP}$ dismissal and increases BRCA1 transcription from BRCA1 promoters. The Laplace transform of the pdf $\mathrm{b}(\mathrm{t})$ is provided by $\tilde{b}(s)=\int_{0}^{\infty} e^{-s t} b(t) d t$

The most applicable distribution for our proposed biological system is two dimensional gamma distribution.

Therefore our transform result as $\tilde{b}(s)=\frac{\left(\frac{\gamma}{s+\mu}\right)^{n}}{n}$

It is applied in the reliability function and hence,

$$
\tilde{R}(s)=\frac{(s+\mu)+\lambda(1-\tilde{b}(s+\mu))}{(s+\mu)(s+\gamma+\lambda(1-\tilde{b}(s+\mu))}
$$

The last expression leads to the Hazard rate average

$\mathrm{H}(\mathrm{u})=\frac{1}{\tilde{R}(s)}=\frac{(s+\mu)(s+\gamma+\lambda(1-\tilde{b}(s+\mu))}{(s+\mu)+\lambda(1-\tilde{b}(s+\mu))}$

Concentrating on the deviations in the first two hours, we conclude that the Laplace transform has the major difference comparably with upcoming consecutive hours. This maximum node of increment in Hazard rate average occurs by the rate of change of time with reference to the difference in Laplace transform. 
From the reliability function expression, we can derive the mean lifetime of the affected

$$
\text { person } \quad m_{F}=\tilde{R}(0)=\frac{\mu+\lambda(1-\tilde{b}(\mu))}{\mu(\gamma+\lambda(1-b(\mu)))}
$$

Thus we can calculate the mean life time as the bound of the untreated control.

\section{RESULTS AND IMPLEMENTATION}

Mainly through this mathematical model we compare the effects of Estrogen with Trichostatin with the help of reliability function which is inversely proportional to Hazard rate. Finding the hazard rate for E2 and TSA added with BRCA1, TFF1 and NBR2 promoters helps us to conclude that Estrogen and its kinetics play vital role in dismissal of CtBP which leads to break the linkages in tumor cells and control the cancer cells.

\section{ESTROGEN AND TRICHOSTATIN EFFECTS WITH BRCA1 -}
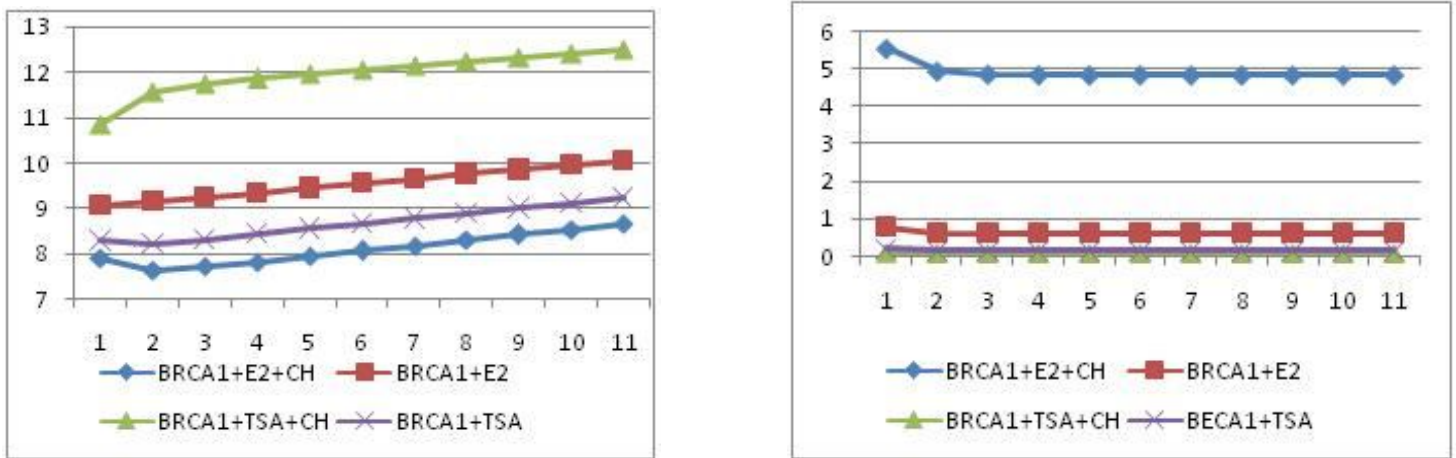

Figure 2(a):Hazard rate average of Estrogen and Trichostatin on BRCA1 promoters

Figure 2(b):Mean life time of Estrogen and Trichostatin on BRCA1 promoters

\section{HAZARD RATE AVERAGE}

Considering the Birth and Death process and hazard rate average curves, we discuss the effects of $\mathrm{E} 2+\mathrm{CH}$ and $\mathrm{TSA}+\mathrm{CH}$ in BRCA1,TFF1 and NBR2 promoters. $\mathrm{E} 2+\mathrm{CH}$ substantially induces BRCA1 neither TFF1 nor NBR2. BRCA1 altered transcription has better inhibition HDAC than untreated control. By the figure 2(a), the effects of E2, TSA, $\mathrm{TSA}+\mathrm{CH}$ on BRCA1 provide sudden and heavy reaction for first two hours and then, hazard rate monotonically increases but Estradiol plus cycloheximide has the mode of influence in first two hours and extends the impact with the next hour and then hazard rate monotonically increases. Therefore Estrogen kinetics inhibits most vigorously in breast cancer and dismisses $\mathrm{CtBP}$ which leads to break the linkages in tumor cells and control rather than Trichostatin. Cycloheximides of E2 and TSA behaves as the bounds for E2 and TSA.

MEAN LIFE TIME

To ensure that the results of Hazard rate average, the Mean life time of $\mathrm{E} 2+\mathrm{CH}$ provides maximum level of monotonically increasing curves.

\section{ESTROGEN AND TRICHOSTATIN EFFECTS WITH TFF1 -}



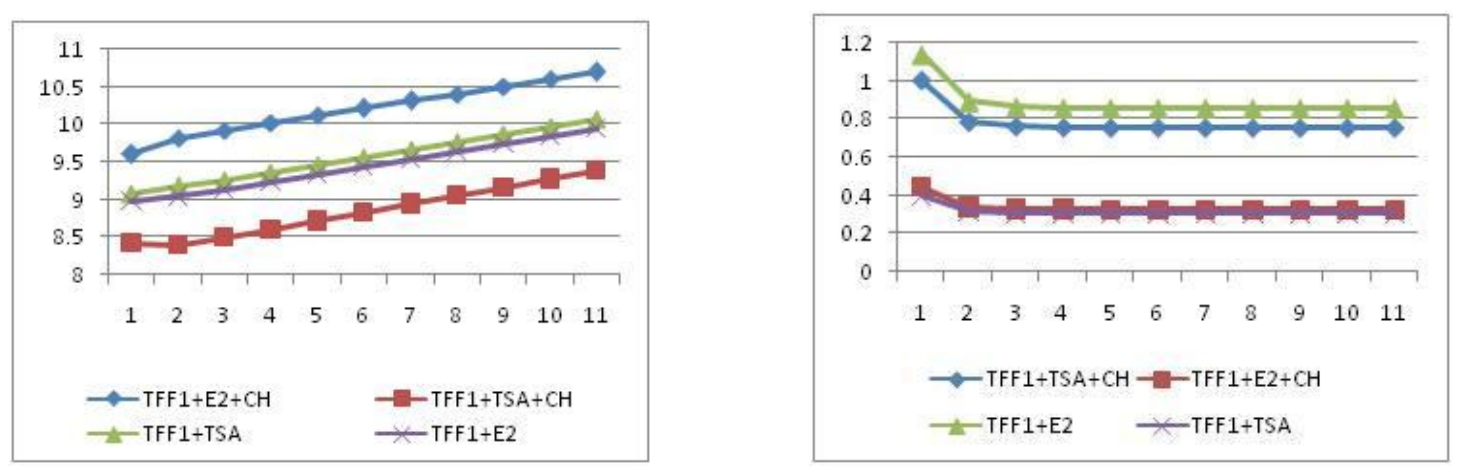

Figure 3(a): Hazard rate average of Estrogen and Trichostatin on trefoil factor 1 Figure 3(b): Mean life time of Estrogen and Trichostatin on trefoil factor 1

\section{HAZARD RATE AVERAGE}

From Figure: 3(a), E2 and its kinetics behaves as the bounds for TSA and its kinetics MEAN LIFE TIME

Mean life time of TSA and TSA+CH behaves as the bound for E2 and $\mathrm{E} 2+\mathrm{CH}$

\section{ESTROGEN AND TRICHOSTATIN EFFECTS WITH NBR2}
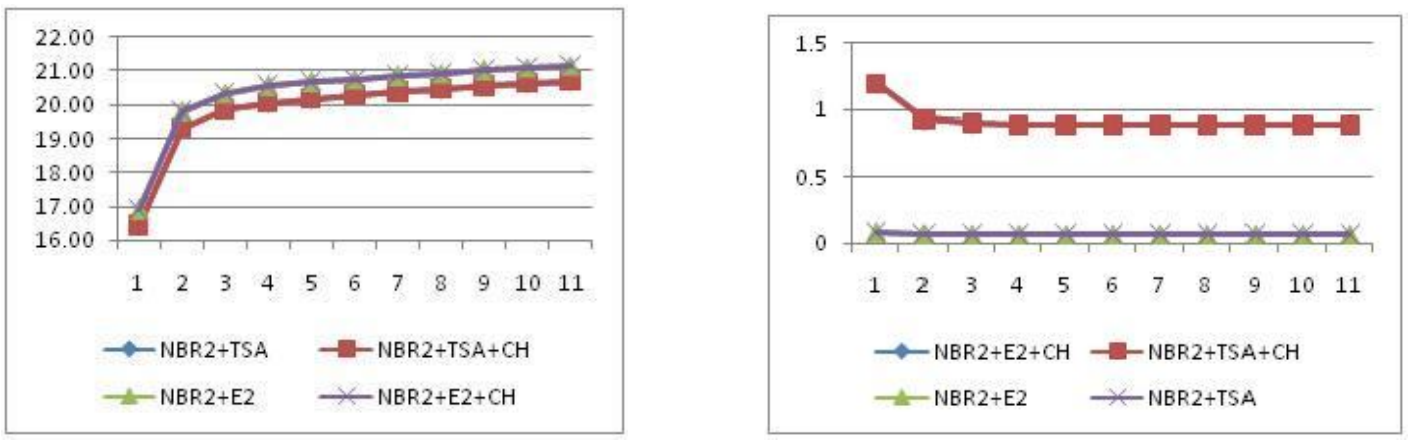

Figure 4(a):Hazard rate average and of Estrogen and TrichostatinA on neibor of BRCA1 gene 2

Figure 4(b):Mean life time of Estrogen and TrichostatinA on neibor of BRCA1 gene 2

\section{HAZARD RATE AVERAGE}

Figure 4(a) and 4(b) show that NBR2 promoters have the same effects between Trichostatin kinetics and Estrogen kinetics which clearly states that according to NBR2 both Estrogen and Trichostatin behaves similar.

MEAN LIFE TIME

NBR2 promoters have the same effects between TSA kinetics and Estrogen kinetics which ensures that according to NBR2 both Estrogen and Trichostatin behaves similar.

According to Figure: 2(a), 2(b), 3(a), 3(b), 4(a), 4(b), the authors trace that the E2 combinations have the least level of hazard rate compared with TSA combinations on BRCA1 but $\mathrm{E} 2+\mathrm{CH}$ and TSA+CH behave as the bound for E2 and TSA on Trefoil factor 1. Also E2 and TSA have the same effect on neibor of BRCA1 gene 2.

The overall conclusion is that BRCA1 promoters have the great influence with Estrogen kinetics compared with TFF1 and NBR2 promoters

Therefore Estrogen kinetics inhibits most vigorously in breast cancer and dismisses CtBP which leads to break the linkages in tumor cells and control rather than Trichostatin and its kinetics.

\section{CONCLUSION}

Generalized Birth and death processes which are the special class of semi markov processes 
are introduced for degradation modeling for Estrogen kinetics in BRCA1 promoters influence $\mathrm{CtBP}$ dismissal and increases BRCA1 transcription from BRCA1 Promoter. Here, the Birth and death process and Hazard rate average $\mathrm{H}(\mathrm{u})$ plays an important role in finding the effects of $\mathrm{E} 2+\mathrm{CH}$ and $\mathrm{TSA}+\mathrm{CH}$ in BRCA1, TFF1 and NBR2 promoters and the Mean life time results help us to confirm those conclusions.

By the figure 2 (a), the effects of E2, TSA, TSA $+\mathrm{CH}$ on BRCA1 provide sudden and heavy reaction for first two hours and then, hazard rate monotonically increases but E2+CH has the mode of influence in first two hours and extends the impact with the next hour and then hazard rate monotonically increases. Therefore Estrogen kinetics inhibits most vigorously in breast cancer and dismisses CtBP which leads to break the linkages in tumor cells and control rather than Trichostatin.

Therefore the authors do agree with the existing survey that $\mathrm{E} 2+\mathrm{CH}$ substantially induces BRCA1 neither TFF1 nor NBR2 ${ }^{[9]}$. We extend this result that BRCA1 altered transcription has better inhibition in HDAC than untreated control and the Estrogen kinetics inhibits most vigorously in breast cancer and dismisses CtBP which leads to break the linkages in tumor cells and control rather than Trichostatin. Incubation of MCF-7 cells with TrichostatinA produces a rapid induction of BRCA1 transcription that occurs earlier than E2. Also we extend that E2 and TSA behaves as the bound for their cycloheximides ; Estrogen and Trichostatin has the same effect on neibor of BRCA1 gene 2.

\section{REFERENCES}

[1] B.Dimitrov1, D.Green, V. Rykov and P.Stanchev. Reliability Model for Biological Objects. In: Longevity, Aging and Degradation Models. Transactions of the First Rus,swn-R'ench Conference (LA 0-2004). Saint Petersburg . June 7-9. 2004, Ed. by V. Antonov. C. Hither. M. Nikulin, V. Polisehook. Saint Petersburg State Politechniral University. SUB. 2004. Vol. 2, pp. 230-210.

[2] B.Dimitrov1, V. Rykov and P.Stanchev(2002) On Multi-State Reliability Systems. In: Proceedings MAlE2002. Trondheitn (Norway) June 17-21, 2002.

[3] Chinnadurai, G. The transcriptional co repressor CtBP: a foe of multiple tumor suppressors. Cancer Res. 69, 731-734 (2009).

[4] D.McDonald, On semi-Markov and semi-regenerative processes. I. 11.1/Z fur Wa/trek, acne. tieS., 42(1978), No. 2, pp.261-377; Ann. Of Pros., 6(1978), No.6, 995-1014.

[5] Fan, S. et al. p300 Modulates the BRCA1 inhibition of estrogen receptor activity. Cancer Res. 62, 141151 (2002).

[6] Fjeld, C.C., Birdsong, W.T. \& Goodman, R.H. Differential binding of NAD ${ }^{+}$and NADH allows the transcriptional co repressor carboxyl-terminal binding protein to serve as a metabolic sensor. Proc. Natl. Acad. Sci. USA 100, 9202-9207 (2003).

[7] Ford, D. et al. Genetic heterogeneity and penetrance analysis of the BRCA1 and BRCA2 genes in breast cancer families. The Breast Cancer Linkage Consortium. Am. J. Hum. Genet. 62, 676-689 (1998).

[8] Landis, S.H., Murray, T., Bolden, S. \& Wingo, P.A. Cancer statistics, 1999. CA Cancer J. Clin. 49, 831 (1999).

[9] Li-Jun Di, Alfonso G Fernandez, Adriana De Siervi, Dan L Longo \& Kevin Gardner. Transcriptional regulation of BRCA1 expression by a metabolic switch Nature Structural \& Molecular Biology17,1406-1413(2010)

[10] Meng, A.X. et al. Hypoxia down-regulates DNA double strand break repair gene expression in prostate cancer cells. Radiother. Oncol. 76, 168-176 (2005).

[11] Pelden S, Insawang T, Thuwajit C, Thuwajit P, The trefoil factor 1 (TFF1) protein involved in doxorubicin-induced apoptosis resistance is upregulated by estrogen in breast cancer cells. Oncol Rep 2013 Sep;30(3):1518-26. doi: 10.3892/or.2013.2593. Epub 2013 Jul 5.

[12] Rodríguez-Enriquez, S. et al. Control of cellular proliferation by modulation of oxidative phosphorylation in human and rodent fast-growing tumor cells. Toxicol. Appl. Pharmacol. 215,208-217 (2006).

[13] Turner, N.C. et al. BRCA1 dysfunction in sporadic basal-like breast cancer. Oncogene 26, 2126-2132 (2007).

[14] V.Rykov. E.Buldaeva On Reliability control of fault tolerance units: regenerative approach. In: Transactions of XXIV International seminar on Stability Problems for Stochastic Mocks, September 111117.2001.Jurmala. Latvia. Transport and Telecommunication Institute. Riga. Latvia, 2004.

[15] V.S.Korolyuk, A.F.Turbin. semi-Markov processes and their applications. Kiev: "Naukova dumka”.1976,1S4p.(in Russian) 
[16] V.S.Korolyuk, A.F.Turbin. Phase aggregation of complex systems. Kiev: "Vish shkola". 1978, 108p.(in Russian)

[17] Vigushin DM, Ali S, Pace PE, Mirsaidi N, Ito K, Adcock I, Coombes RCTrichostatin A is a histone deacetylase inhibitor with potent antitumor activity against breast cancer in vivo,Cin cancer Res. 2001 Apr;7(4):971-6.

[18] W.Feller. An Introduction to Probability Theory and its Applications V. II John Wiley \& Sons, Inc. N.Y.-Lnd.-Sidney, 1966.

[19] Wilson, C.A. et al. Localization of human BRCA1 and its loss in high-grade, non-inherited breast carcinomas. Nat. Genet. 21, 236-240 (1999).

[20] Zhang, Q., Piston, D.W. \& Goodman, R.H. Regulation of corepressor function by nuclear NADH. Science 295, 1895-1897 (2002). 